\title{
INTENSIVE THERAPY WITH INSULIN PUMP IN TYPE 1 DIABETES MELLITUS
}

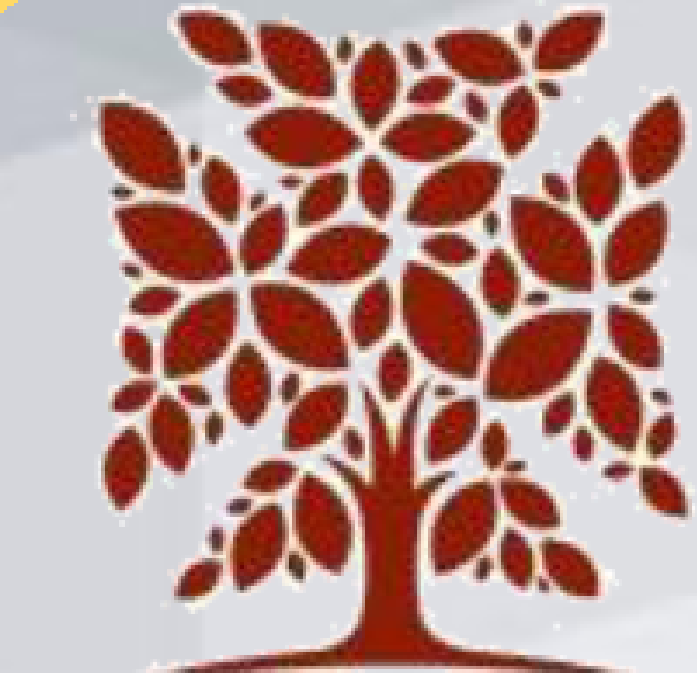

SÃO JOÃO

Gouveia $\mathrm{I}^{3}$, Neves $\mathrm{C}^{1,3}$, Oliveira $\mathrm{SC}^{1,3}$, Neves $\mathrm{JS}^{1,3}$, Dias $\mathrm{C}^{2}$, Carvalho $\mathrm{D}^{1,3,4}$

${ }^{1}$ Departmente of Endocrinology, Diabetes and Metabolism;

${ }^{2}$ Department of Health Information and Decision Sciences; São João Hospital Centre.

${ }^{3}$ Faculty of Medicine, University of Porto

${ }^{4}$ Institute for Research and Innovation in Health Sciences of the Faculty of Medicine of the University of Porto

\section{Background}

Continuous subcutaneous insulin infusion (CSII), alongside multiple daily injections (MDI) are the most frequent therapeutic modalities of type 1 Diabetes Mellitus (DM).

\section{Objectives}

We aimed to evaluate the effects of the transition from $\mathrm{MDI}$ to CSII in the treatment of type $1 \mathrm{DM}$.

\section{Matherial and Methods}

This retrospective longitudinal study analyzed patients that received treatment with CSII from 2006 to 2014 . We evaluated values such as weight, $\mathrm{HbA1c}$, serum glycose, lipid profile, creatinine, weekly frequency of hypoglycemias $(<70 \mathrm{mg} / \mathrm{dL})$ and hyperglycemia $(>200 \mathrm{mg} / \mathrm{dL})$ as well the presence of microvascular complications. The effects of CSII were compared according to the following subgroups: HbA1c pre-CSII $(\leq 7.0 \%$ vs $>7.0 \%)$; age ( $\leq 35$ years vs $>35$ years); sex distribution; duration of disease ( $\leq 15$ years vs $>15$ years); presence of microvascular complications.

\section{Results}

\section{$\mathrm{N}=85$ patients}

- $58.8 \%$ women, medium age of $37 \pm 11$ years and medium duration of disease of $15 \pm 9$ years.

$\downarrow$ Weekly frequency of episodes of hypo and hyperglycemia

- Hypoglycemia $\rightarrow 3.0$ (1.5-6.0) vs 2.0 (1.0-3.9); $p=0.001$

- Hyperglycemia $\rightarrow 5.5$ (3.0-7.0) vs 2.5 (1.8-4.5); $p<0.001$

\section{Conclusion}

The CSIl therapy is more effective than MDI as demonstrated by the significant reduction of hypoglycemia and hyperglycemia episodes. We also observed benefits in the reduction of $\mathrm{HbA} 1 \mathrm{c}$ with the CSII treatment in the $\mathrm{HbA} 1 \mathrm{c}>7.0 \%$ subgroup (worst metabolic control).

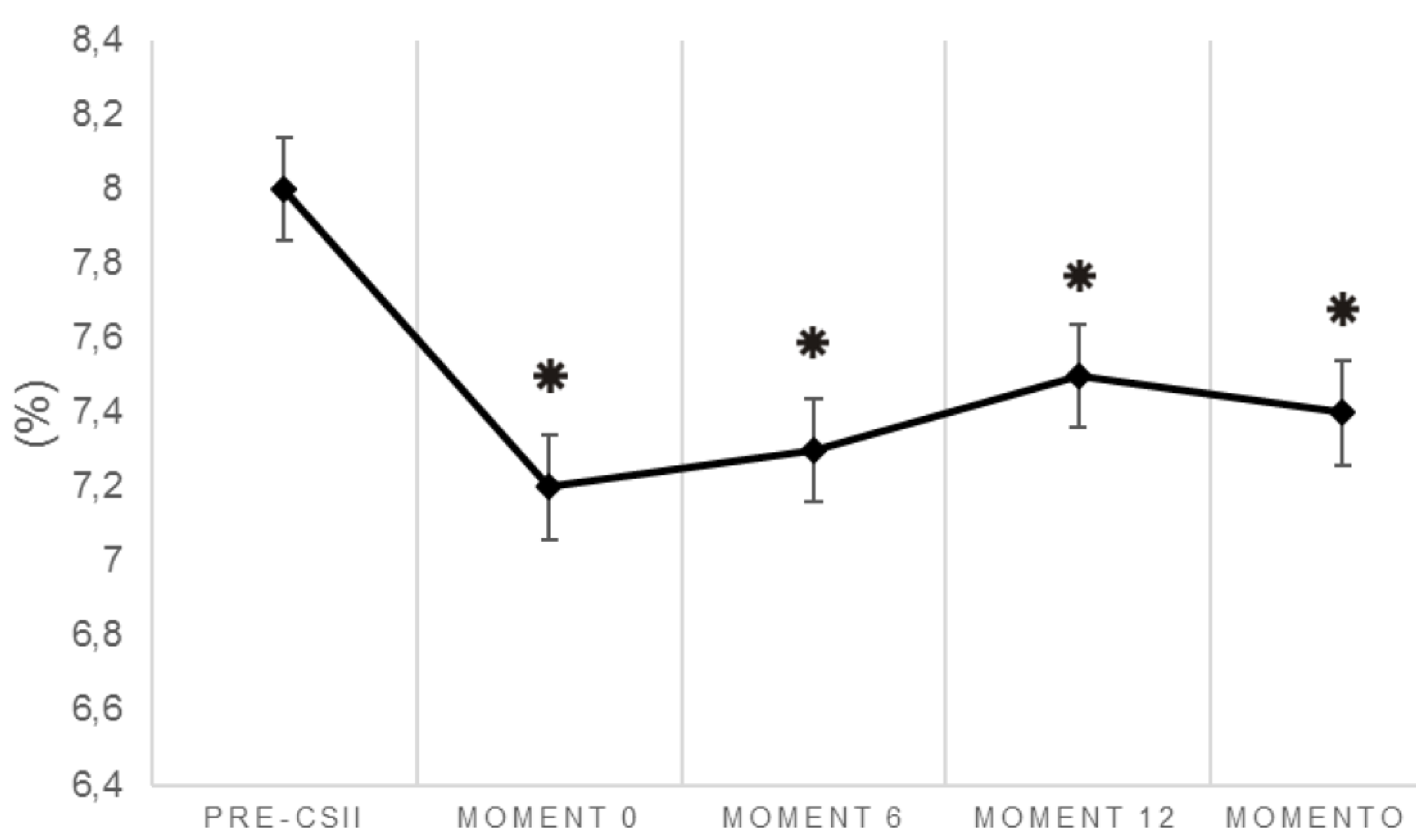

Figure 1. Median $\mathrm{HbA1c}(\%)$ values through the moments of the study. $\quad * p<0.001$ in comparison with MDI regimen value (Pre-CSII)

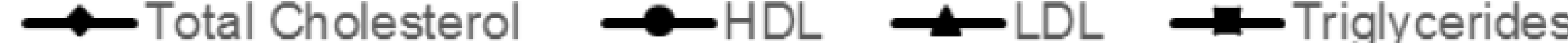

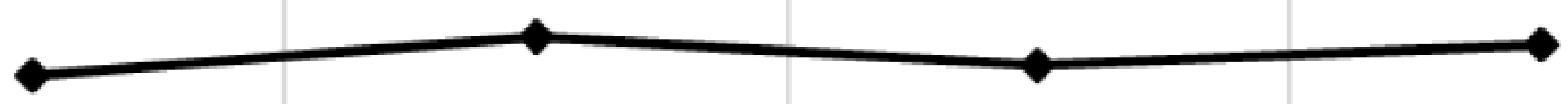

150

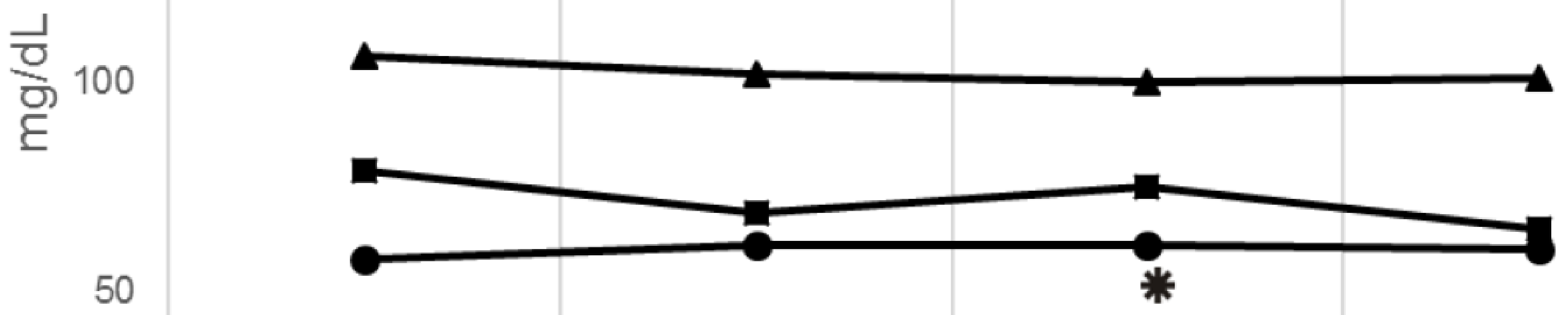

MOMENT

MOMENT

MOMENT

MOMENT 24

Figure 2. Median value variation of lipid profile through the moments of the study. $* p<0.05$ in comparison with moment zero. 